\title{
Formation of Rosette-Shaped Cd(II) Thiolate Coordination Polymer in Aqueous Solution and Conversion to CdS by Calcination
}

\author{
Sungjune Han, ${ }^{\dagger}$ Myunghan Lee, ${ }^{\dagger}$ and Young Jin Jeon ${ }^{*}$ \\ Department of Applied Chemistry, College of Natural Sciences, Konkuk University, Chungju 380-701, Korea \\ *E-mail:jeonyj@kku.ac.kr \\ ${ }^{\dagger}$ Department of Advanced Technology Fusion, Konkuk University, Seoul 143-701, Korea \\ Received February 2, 2010, Accepted February 19, 2010
}

Key Words: Coordination polymer, Morphology control, Cadmium sulfide, Calcination, Metal thiolate

\begin{abstract}
Coordination polymers $(\mathrm{CPs})^{1-3}$ have attracted considerable attention because of their potential applications in gas storage, ${ }^{4-6}$ catalysis, ${ }^{7}$ ion exchange, ${ }^{8}$ separation, ${ }^{9}$ biomedicine, ${ }^{10,11}$ etc. For use in the abovementioned applications, the structure and morphology of these CPs have been controlled by judicious choice of metals, ligands, and reaction conditions. Recently, Oh and coworkers have reported that CPs can be successfully converted into metal oxides by calcination without causing any significant change in their morphology. ${ }^{12,13}$ This strategy offers great opportunities to CPs chemistry that various metal oxides can be formed as morphologies of CPs dictate. The CPs synthesized thus far mainly comprise transition metal ions and organic ligands having more than two carboxylate functional groups. The synthesized CPs are calcined to form metal oxides, while maintaining the shape of the particles of the former. Many of the important compound semiconductors are metal chalcogenides formed between a metal and sulfur. Yamamoto's group has reported that (alkylthiolato)metal complexes can be pyrolyzed to form metal sulfides. ${ }^{14}$ The same strategy can be applied to the preparation of metal sulfide nanostructures and microstructures. Metal sulfides are synthesized as follows. Metal ions are allowed to react with ligands containing sulfur sources, such as organothiols, to form metal sulfide CPs, which are subsequently calcined to obtain metal sulfide nanostructures or microstructures. This is expected to be a novel method for obtaining metal sulfide nanostructures or microstructures via the formation of metal thiolate CPs. Herein, we report the formation of CPs from $\mathrm{Cd}$ (II) ions and mercaptosuccinic acid (MSA) in aqueous solution and the calcination of the obtained CPs to form $\mathrm{CdS}$ particles without any change in the morphology.
\end{abstract}

\section{Experimental Section}

All the chemicals were analytical grade and were used as received without further purification. Distilled water was purified by using a Millipore Milli-Q water purification system (Barnstead, USA). Infrared (IR) spectra were recorded with a MIDAC M series IR spectrometer. Elemental (CHNS) analysis was performed using a Valio EL elemental analyzer at Central Machines and Facilities Shop in POSTECH (Pohang, Korea). Powder X-ray diffraction (XRD) patterns were recorded using a Philips X'pert-MPD diffractometer (Philips Analytical, Netherlands) with a $\mathrm{Cu} \mathrm{K} \alpha$ radiation source $(\lambda=1.54 \AA)$ The operat- ing voltage and current were $40 \mathrm{kV}$ and $35 \mathrm{~mA}$, respectively. Calcination was performed in a nitrogen atmosphere in a tube furnace (Daemyoung Inc., Korea) equipped with a Yokogawa UP350E temperature control unit. Scanning electron microscopy (SEM) and energy-dispersive X-ray spectroscopy (EDX) measurements were performed using a JEOL JSM 6700 scanning electron microscope. Raman spectra were recorded using an in Via Raman spectrometer (Renishaw, USA) at an excitation wavelength of $488 \mathrm{~nm}$.

Synthesis of coordination polymer. In a typical procedure, aqueous solutions of $\mathrm{Cd}\left(\mathrm{NO}_{3}\right)_{2} \cdot \mathrm{xH}_{2} \mathrm{O}(10 \mathrm{mM}, 5 \mathrm{~mL})$ and mercaptosuccinic acid (MSA, $100 \mathrm{mM}, 5 \mathrm{~mL}$ ) were mixed in a vial at room temperature. The $\mathrm{pH}$ of the resulting mixture was found to be approximately 3 . Within a day, white particles began to precipitate from the mixture. At each time point, the precipitated particles were separated by centrifugation, washed with distilled water and ethanol, and then dried in vacuum. IR ( $\mathrm{KBr}$ pellet, $\mathrm{cm}^{-1}$ ) 3563.8br, 2921.6w, 2360.3w, 1913.0w, 1693.2s, 1573.6s, $1429.0 \mathrm{~s}, 1220.7 \mathrm{~s}, 987.4 \mathrm{~m}, 881.3 \mathrm{~s}, 730.9 \mathrm{~s}, 621.0 \mathrm{~m}$. Anal. Cald. for $\left[\mathrm{Cd}\left(\mathrm{C}_{4} \mathrm{H}_{5} \mathrm{O}_{4} \mathrm{~S}\right)\right]_{\mathrm{n}}$ : C, 18.37; H, 1.93; S, 12.26. Found: $\mathrm{C}$, 18.32; H, 2.04; S, 12.32 .

Preparation of CdS by calcination of CPs. The abovementioned CP sample was placed in an alumina boat in a tube furnace and calcined in a nitrogen atmosphere. The temperature elevation was programmed using the Yokogawa UP350E temperature control unit as follows: temperature was elevated from room temperature to $400{ }^{\circ} \mathrm{C}$ for $30 \mathrm{~min}$ and was maintained at the temperature for the desired time $(1 \mathrm{~h}$ and $3 \mathrm{~h})$. IR $(\mathrm{KBr}$ pellet, $\mathrm{cm}^{-1}$ ) $1614.1 \mathrm{w}, 1432.9 \mathrm{br}, 1118.5 \mathrm{~s}, 858.2 \mathrm{w}, 619.0 \mathrm{~s}$.

\section{Results and Discussion}

To verify whether metal thiolate CPs could be converted to metal sulfide nanostructures or microstructures by calcination, we chose $\mathrm{Cd}$ (II) as the metal ion and MSA as the sulfur source. We attempted to synthesize CPs from Cd(II) and MSA and study the morphology of the resulting product. First, we mixed Cd(II) ions and MSA (molar ratio: 1:10) in aqueous medium in a vessel and waited for 3 days until white precipitates were formed.

The SEM images in Figures 1a and $1 \mathrm{~b}$ show that the CPs particles are micron sized and rosette shaped. The size of the particles is estimated to be approximately $87 \pm 15 \mu \mathrm{m}$. Elemental analysis shows that the CPs contain Cd(II) and deprotonated 

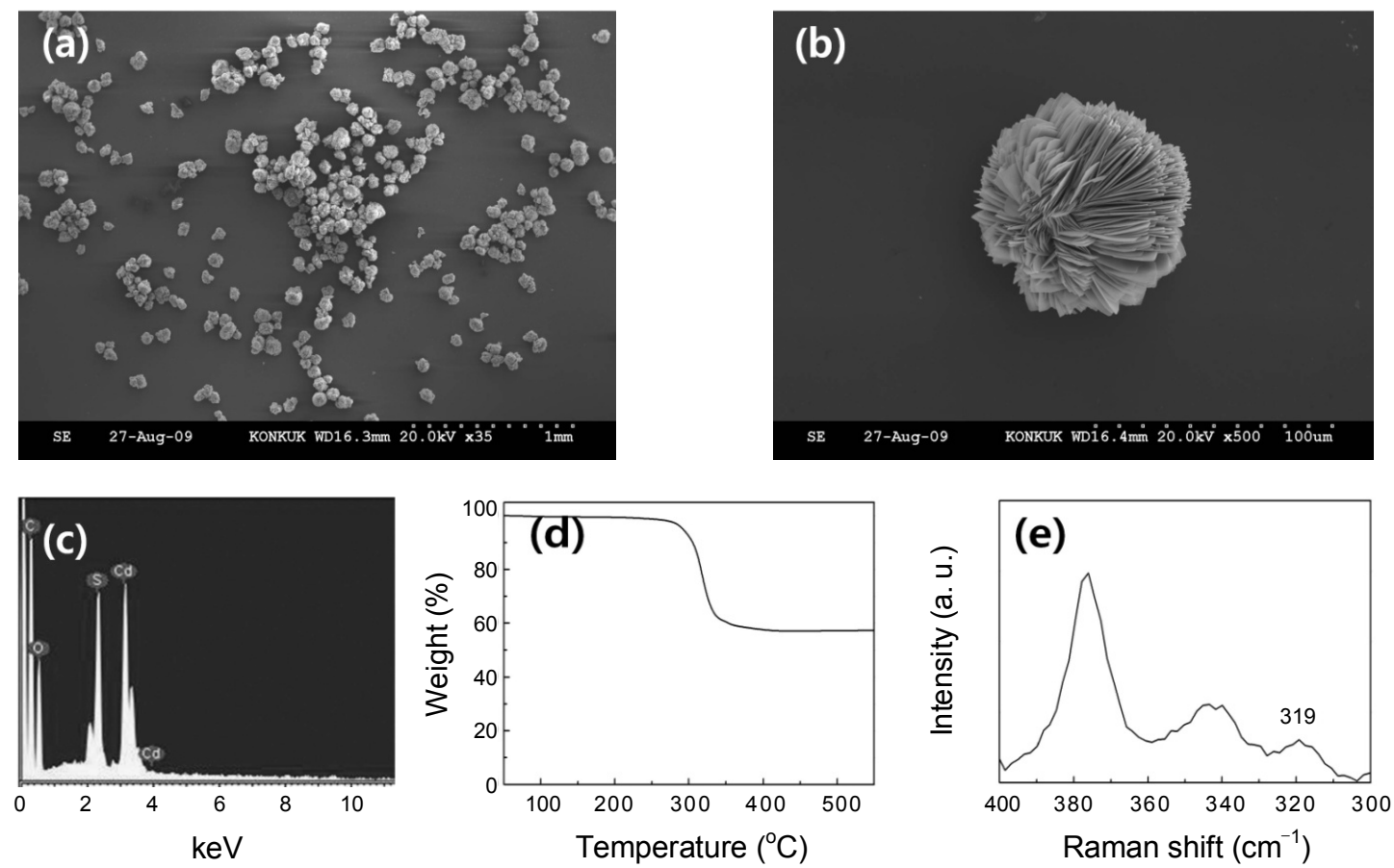

Figure 1. (a) and (b) SEM images, (c) EDX spectrum, (d) TGA curve, and (e) Raman spectrum of Cd-MSA CPs.
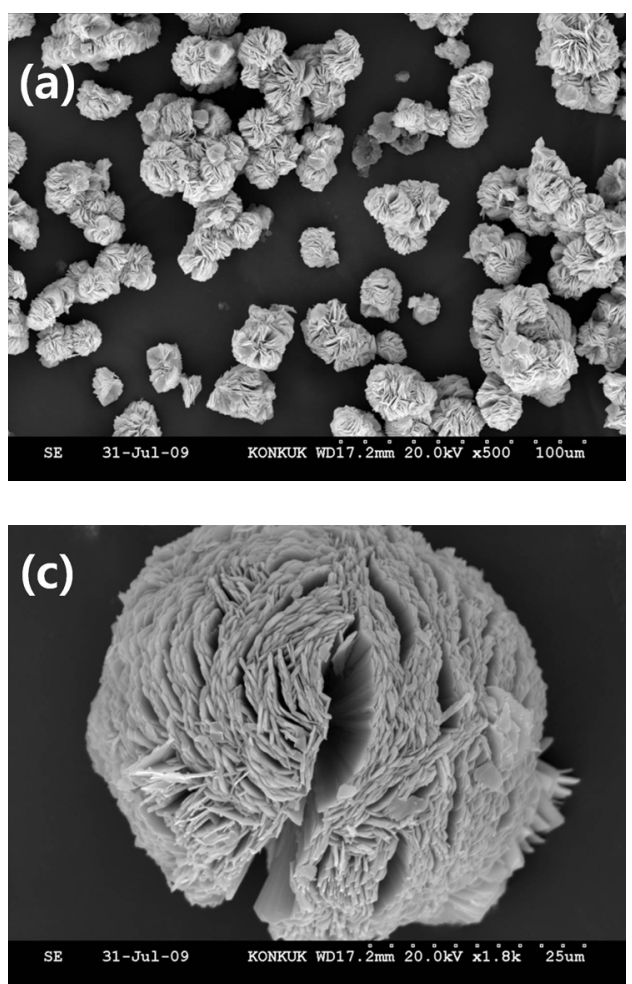
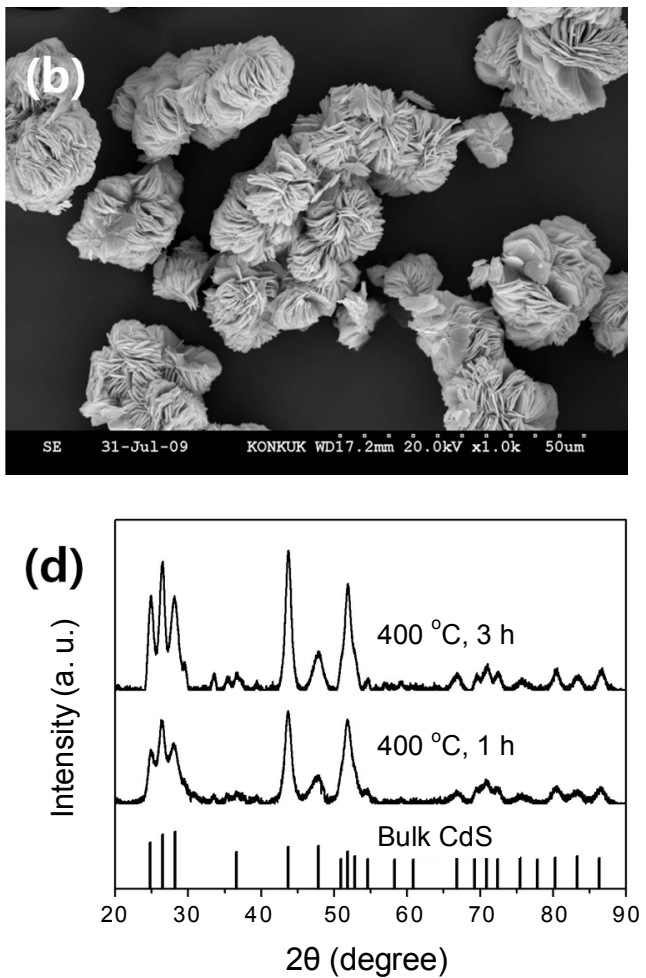

Figure 2. (a)-(c) SEM images of CdS microstructures produced by calcination of Cd-MSA at $400{ }^{\circ} \mathrm{C}$ for $1 \mathrm{~h}$; (d) XRD pattern of bulk CdS (JCPDS No. 41-1049) and the CdS calcined at $400{ }^{\circ} \mathrm{C}$ for $1 \mathrm{~h}$ and $3 \mathrm{~h}$.

MSA in a 1:1 molar ratio; this value is in good agreement with that reported in the literature. ${ }^{15}$ The EDX data (Figure 1c) show that the atomic percent ratio of $\mathrm{Cd}$ to $\mathrm{S}$ is around 4.78: 4.44, which is quite close to $1: 1$. Thermogravimetric analysis (TGA) of the CPs reveals a weight loss of approximately $44 \%$, which supports the CP molar ratio estimated by elemental analysis and EDX. The Cd-MSA CPs are stable up to approximately $300{ }^{\circ} \mathrm{C}$ (Figure 1d). The overall Cd:MSA molar ratio in the CPs is $1: 1$, although the two components are initially mixed in a $1: 10$ molar ratio. Detailed analysis of the molecular arrangement in the Cd-MSA CPs is difficult because of interference from weak peaks present in the powder XRD spectrum and the poly- 


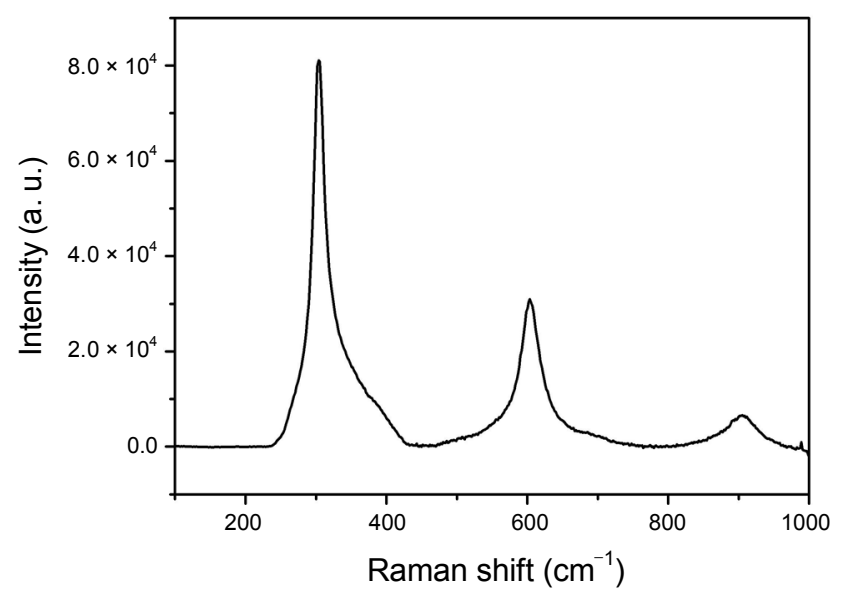

Figure 3. Raman spectrum of CdS produced by calcination of Cd-MSA $\mathrm{CP}$ microstructures.

crystalline nature of the particles. Other studies have also reported the inherent difficulties involved in determining the exact molecular structure of CPs. ${ }^{16,17}$ Nevertheless, the band at 319 $\mathrm{cm}^{-1}$ in the Raman spectrum corresponds to the formation of $\mathrm{Cd}-\mathrm{O}$ and $\mathrm{Cd}-\mathrm{S}$ bonds; this confirms the extended coordination of the $\mathrm{O}$ and $\mathrm{S}$ atoms of MSA to the $\mathrm{Cd}(\mathrm{II})$ ion (Figure 1e). ${ }^{18}$

The formation of the $\mathrm{Cd}$ thiolate complex indicates the possible conversion of the complex into $\mathrm{CdS}$ particles by calcination. Calcination is performed in a nitrogen atmosphere to prevent the oxidation of CdS. Figures $2 \mathrm{a}-\mathrm{c}$ are SEM images obtained after calcinations of the CPs at $400{ }^{\circ} \mathrm{C}$ for $1 \mathrm{~h}$, which indicate the formation of CdS particles. The morphology of the obtained CdS particles is identical to that of the CPs; however, the diameter of the CdS particles is only $35 \%$ of that of the $\mathrm{CP}$ particles. The diameter of the CdS particles is estimated to be approximately $22 \mu \mathrm{m}$. Such a decrease in the particle size upon calcination has also been observed in previous studies concerning metal oxide formation. ${ }^{12}$ Moreover, in the present study, no particle aggregation is observed during the calcination. The presence of MSA in Cd-MSA and the elimination of MSA from $\mathrm{CdS}$ is confirmed by IR experiments (Experimental Section). As seen in the IR spectrum, the characteristic carboxylic acid peaks disappear after calcination.

The calcined CdS particles are characterized by powder XRD and Raman spectroscopy. The XRD results reveal that $\mathrm{CdS}$ has a hexagonal würzite phase, and there is no evidence for the presence of any cubic phase (JCPDS No. 41-1049) (Figure 2d). Interestingly, despite the presence of oxygen atoms in the carboxylate groups of MSA, CdO formation is not observed. Peaks are broad compared to those of bulk $\mathrm{CdS}$, which is indicative of small crystal size although the CdS particles are over micrometer in size. The grain size of $\mathrm{CdS}$ is calculated by using the Debye-Scherrer equation $\mathrm{D}=0.9 \lambda / \mathrm{B} \cos \theta$, where $\mathrm{D}$ is the average crystal size; $\lambda$, the wavelength of the X-rays used; $\mathrm{B}$, the angular line width of half-maximum intensity; and $\theta$, the diffraction angle in degrees. ${ }^{19}$ The size of the $\mathrm{CdS}$ particles is estimated to be approximately $7.8 \mathrm{~nm}$. Therefore, we infer that the CdS microparticles are composed of $7.8 \mathrm{~nm}$ nanocrystals.

Figure 3 shows the Raman spectrum of the calcined CdS microstructure. Characteristic bands corresponding to $\mathrm{CdS}$ are identified in the spectrum. The three bands at $304 \mathrm{~cm}^{-1}, 604$ $\mathrm{cm}^{-1}$, and $905 \mathrm{~cm}^{-1}$ correspond to the first-, second-, and thirdorder transverse optical (TO) phonon modes of the CdS CPs, respectively. ${ }^{20}$ Further, these bands are shifted to a lower frequency as compared to the bulk CdS bands, indicating that the $\mathrm{CdS}$ particles are smaller than the corresponding bulk particles.

\section{Conclusion}

We have synthesized rosette-shaped Cd-MSA CPs by a reaction between $\mathrm{Cd}(\mathrm{II})$ ions and MSA in aqueous solution and calcined the obtained CPs to obtain CdS microstructures. Upon calcination, the morphology of the CPs does not undergo any significant change, but the particle diameter decreases by $74 \%$. This indicates that our strategy can be used for the synthesis of CPs from other metal thiolates as well. We expect this strategy to be suitable for the preparation of important metal chalcogenide nanostructures and microstructures that can be used in future applications.

Acknowledgments. This study was supported by the Faculty Research Fund of Konkuk University in 2009.

\section{References and Notes}

1. Spokoyny, A. M.; Kim, D.; Sumrein, A.; Mirkin, C. A. Chem. Soc. Rev. 2009, 38, 1218-1227.

2. Oh, M.; Mirkin, C. A. Nature 2005, 438, 651-654.

3. Lin, W.; Rieter, W. J.; Taylor, K. M. Angew. Chem. Int. Ed. 2009, 48, 650-658.

4. Yaghi, O. M.; O'Keeffe, M.; Ockwig, N. W.; Chae, H. K.; Eddaoudi, M.; Kim, J. Nature 2003, 423, 705-714.

5. Zhao, X.; Xiao, B.; Fletcher, A. J.; Thomas, K. M.; Bradshaw, D.; Rosseinsky, M. J. Science 2004, 306, 1012-1015.

6. Koh, K.; Wong-Foy, A. G.; Matzger, A. J. J. Am. Chem. Soc. 2009, 131, 4184-4185.

7. Seo, J. S.; Whang, D.; Lee, H.; Jun, S. I.; Oh, J.; Jeon, Y. J.; Kim, K. Nature 2000, 404, 982-986.

8. Oh, M.; Mirkin, C. A. Angew. Chem. Int. Ed. 2006, 45, 5492-5494.

9. Kosal, M. E.; Chou, J. H.; Wilson, S. R.; Suslick, K. S. Nat. Mater. 2002, 1, 118-121.

10. Rieter, W. J.; Pott, K. M.; Taylor, K. M.; Lin, W. J. Am. Chem. Soc. 2008, 130, 11584-11585.

11. Horcajada, P.; Chalati, T.; Serre, C.; Gillet, B.; Sebrie, C.; Baati, T.; Eubank, J. F.; Heurtaux, D.; Clayette, P.; Kreuz, C.; Chang, J. S.; Hwang, Y. K.; Marsaud, V.; Bories, P. N.; Cynober, L.; Gil, S.; Ferey, G.; Couvreur, P.; Gref, R. Nat. Mater. 2009, 9, 172-178.

12. Jung, S.; Cho, W.; Lee, H. J.; Oh, M. Angew. Chem. Int. Ed. 2009, 48, 1459-1462.

13. Cho, W.; Lee, Y. H.; Lee, H. J.; Oh, M. Chem. Commun. 2009 , 4756-4758.

14. Osakada, K.; Yamamoto, T. Inorg. Chem. 1991, 30, 2328-2332.

15. Crisponi, G.; Diaz, A.; Nurchi, V. M.; Pivetta, T.; Estevez, M. J. T. Polyhedron 2002, 21, 1319-1327.

16. Cho, W.; Lee, H. J.; Oh, M. J. Am. Chem. Soc. 2008, 130, 1694316946.

17. Anokhina, E. V.; Vougo-Zanda, M.; Wang, X.; Jacobson, A. J. J. Am. Chem. Soc. 2005, 127, 15000-15001.

18. Ramos, J. M.; Faget, O. G.; Felcman, J.; Tellez, S. C. Spectrochim. Acta A Mol. Biomol. Spectrosc. 2008, 71, 1364-1370.

19. Nanda, J.; Sapra, S.; Chandrasekharan, N.; Hodes, G. Chem. Mater. 2002, 12, 1018-1024.

20. Xiong, S.; Xi, B.; Wang, C.; Zou, G.; Fei, L.; Wang, W.; Qian, Y. Chem. Eur. J. 2007, 13, 3076-3081. 